\title{
Análise, processamento e prognose de falhas em motores de combustão (otto) através de vibração: aplicação de sistemas imunológicos artificiais
}

\author{
Analysis, processing and prognosis of faults in combustion engines (otto) through vibration: \\ application of artificial immune systems \\ Análisis, procesamiento y pronósticos de fallas en motores de combustión (otto) a través de \\ vibración: aplicación de sistemas inmunes artificiales
}

Gustavo Henrique Gonçalves

ORCID: https://orcid.org/0000-0002-0288-4423 Faculdade de Tecnologia de Araçatuba, Brasil

E-mail: gustavo.goncalves6@fatec.sp.gov.br

Roberto Outa

ORCID: https://orcid.org/0000-0002-8649-1722

Faculdade de Tecnologia de Araçatuba, Brasil E-mail: roberto.outa@fatec.sp.gov.br

Fabio Roberto Chavarette

ORCID: https://orcid.org/0000-0002-1203-7586 Universidade Estadual Paulista, Brasil E-mail: fabio.chavarette@unesp.br Aparecido Carlos Gonçalves

ORCID: https://orcid.org/0000-0001-5376-3392 Universidade Estadual Paulista, Brasil E-mail: aparecido.carlos@unesp.br

Adriana Garcia

ORCID: https://orcid.org/0000-0003-3054-1804 Institute of Technological Research of the State of São Paulo, Brasil E-mail: adrianag@ipt.br

Paulo Sérgio Barbosa dos Santos ORCID: https://orcid.org/0000-0001-8211-3882 Universidade Estadual Paulista, Brasil Email: paulo.sb.santos@unesp.br

\begin{abstract}
Resumo
Este trabalho demonstra a aplicação dos sistemas imunológicos artificiais (AIS) de seleção negativa na prognose e detecção de falhas nas misturas de combustíveis. A motivação deste estudo está relacionada à manutenção de motores de combustão ciclo OTTO, cuja propriedade difere das análises físico-químicas de laboratórios especializados. Este trabalho foi dividido em fases distintas, sendo elas, a execução do experimento considerando um motor de motocicleta; a coleta de sinal e formação do banco de dados considerando o combustível de posto (500ml); o combustível de posto $(500 \mathrm{ml}) \mathrm{com} 100 \mathrm{ml}$ de etanol de posto; o combustível de posto $(500 \mathrm{ml}) \mathrm{com} 200 \mathrm{ml}$ de etanol de posto. O resultado encontrado dos diferentes sinais, após a aplicação do AIS, demonstrou com sucesso o grupamento e classificação dos sinais dos bancos de dados.
\end{abstract}

Palavras-chave: Biossistemas; Vibração; Sistemas imunológicos artificiais; Motores de combustão; SHM.

\begin{abstract}
This work demonstrates the application of artificial immunological systems (AIS) of negative selection in prognosis and detection of failures in fuel mixtures. The motivation of this study is related to the maintenance of OTTO cycle combustion engines, whose property differs from the physicochemical analyses of specialized laboratories. This work was divided into distinct phases, which were the execution of the experiment considering a motorcycle engine; signal collection and database formation considering gas station fuel $(500 \mathrm{ml})$; the gas station fuel $(500 \mathrm{ml})$ with $100 \mathrm{ml}$ of gas station ethanol; the gas station fuel $(500 \mathrm{ml})$ with $200 \mathrm{ml}$ of gas station ethanol. The result found of the different signals, after the application of the AIS, successfully demonstrated the grouping and classification of the signals of the databases.
\end{abstract}

Keywords: Biosystems; Vibration; Artificial immune systems; Combustion engines; SHM. 


\begin{abstract}
Resumen
Este trabajo demuestra la aplicación de sistemas inmunológicos artificiales (AIS) de selección negativa en pronóstico y detección de fallos en las mezclas de combustible. La motivación de este estudio está relacionada con el mantenimiento de motores de combustión de ciclo OTTO, cuya propiedad difiere de los análisis fisicoquímicos de laboratorios especializados. Este trabajo se dividió en fases distintas, que fueron la ejecución del experimento considerando un motor de motocicleta; recolección de señales y formación de bases de datos teniendo en cuenta el combustible de la gasolinera $(500 \mathrm{ml})$; el combustible de la gasolinera $(500 \mathrm{ml})$ con $100 \mathrm{ml}$ de etanol de la gasolinera; el combustible de la gasolinera $(500 \mathrm{ml})$ con $200 \mathrm{ml}$ de etanol de gasolinera. El resultado encontrado de las diferentes señales, después de la aplicación del AIS, demostró con éxito la agrupación y clasificación de las señales de las bases de datos.
\end{abstract}

Palabras clave: Biosistemas; Vibración; Sistemas inmunológicos artificiales; Motores de combustión; SHM.

\title{
1. Introduçãa
}

Sistemas de funcionamento complexo como o motor de motocicletas, atuam diferentemente, baseando-se em princípios de indução por faísca e em sistemas elétricos. O funcionamento de um motor de indução a faísca, produz uma faísca internamente a câmara do motor, resultando na explosão da mistura gasosa contendo combustível. A força gerada pela explosão é transferida a uma superfície metálica que aciona um mecanismo, baseado em um sistema de alavanca, transferindo essa energia de rotação ao sistema interligado. Este princípio é comumente chamado de combustão de ciclo Otto (Pulkrabek, 2003).

Os motores de combustão contêm sistemas mecânicos que ao se atritarem produzem reações no próprio sistema, à energia do atrito se propaga na estrutura de forma que o sistema oscila de forma complexa. Este fato pode ser entendido como a energia da vibração se propagando no sistema.

Considerando este fato, observa-se que a energia gerada pela explosão do combustível é transferida ao sistema, cuja energia da vibração ressoa de forma que, para cada tipo de elemento: combustível e componentes mecânicos do sistema altera o sistema, resultando em diferentes formas de vibrar.

As diferentes formas de vibrar de um motor podem gerar ruídos indesejados soa sistema, o qual pode ser ouvido através de diferentes timbres ou frequências. Neste caso, determinadas estratégias podem ser tomadas para o enclausuramento e tratamento do ruído sonoro, pois as normas e regulamentos determinam uma condição desejada de ruído sonoro em um determinado ambiente. É possível entender estes princípios através de alguns trabalhos de pesquisa, como por exemplo: a aplicação de controle de ruído acústico e vibracional em motores de combustão, baseando-se na melhora da qualidade através de aplicações em materiais e design de pneus (Bein, et al., 2012). Um outro grupo de pesquisadores demonstram que através de simulação computacional é possível controlar a emissão de ruídos e melhorar o processo de desenvolvimento de protótipos considerando estudos de fluidos dinâmicos, mecanismos estruturais, e termodinâmica (Gabbert et al., 2017). Outro grupo demonstra já um resultado de detecção de falhas em sistemas rotativos, utilizando a teoria de vibração e o sistema imunológico artificial de seleção negativa (Outa et al., 2020).

Este trabalho tem como objetivo detectar os sinais de diferentes tipos de misturas de combustível, utilizando a teoria de vibração aplicada ao sistema imunológico artificial de seleção negativa. O sinal ruidoso é de um motor de combustão de motocicleta e o combustível utilizado é de uso comercial, portanto, a condição experimental, propõe a condição diária de muitos que possuem este meio de locomoção. Ainda na análise do sinal, serão inseridas técnicas da ciência da computação: a mineração de dados demonstrando a separação de grupos dos sinais filtrados.

\section{Monitoramento de Integridade Estrutural}

A indústria 4.0 tem exigido uma reavaliação no conceito de sistemas produtivos, e observa-se que esta condição tem afetado as indústrias de uma forma geral. 
O conceito da indústria 4.0 pode ser considerado com um sistema produtivo, integrado por computadores, dispositivos móveis, sendo todos ligados a uma determinada rede, cujo objetivo é a busca da otimização de informações e ferramentas gerenciais entre os stakeholders, e os clientes internos e externos a empresa (Sacomano et al., 2018).

A formação da indústria 4.0, pode ser feita baseada em três grupos distintos e interligados, são eles: (1) elementos de base, (2) elementos estruturantes, (3) elementos complementares. Os elementos de base são subdivididos em: internet das coisas (IoT); sistemas cyber-físicos; e internet de serviços (IoS). Já os elementos estruturantes correspondem a: automação; comunicação de máquina a máquina (M2M); inteligência artificial (AI); análise de big data; computação na nuvem; integração de sistemas; segurança cibernética. Por fim, os elementos complementares são: etiquetas de RFID; QR code; realidade aumentada (RA); realidade virtual (RV); e manufatura aditiva (Sacomano et al., 2018).

Em especifico a inteligência artificial, na indústria, tem a finalidade de controlar o processo de produção, como também, fornece e sugere informações a diversas áreas ligadas a produção para a tomada de decisões (Sacomano et al., 2018).

A característica de controle de processos de produção por inteligência artificial permite que a manutenção de máquinas e equipamentos possa ser analisada e criterizadas por diferentes métodos de prognose e detecção de falhas. Com isso, as condições de dano e falhas devem ser definidas e entendidas. A palavra dano pode ser entendida como um evento que causa a variação ou comprometimento do desempenho de uma peça ou componente, quando comparado ao seu estado inicial sem danos (Deraemaeker et al, 2010; Farrar et al., 2013). A palavra falha é entendida quando a peça ou componente não executa mais a função especificada em projeto, cujas causas podem estar relacionadas à ação de forças internas ou externas, e até a desestabilidade do sistema (Yan, 2015).

A diagnose de danos e falhas são processos que devem ser implementados em indústrias em geral, para qualquer sistema dinâmico, cujo objetivo é a prevenção do comportamento da peça e preservação da sua função. O monitoramento de integridade estrutural, do inglês structural health monitoring (SHM) é considerado uma estratégica para a identificação de danos em infraestruturas de engenharia espacial, civil e mecânica, e é um conceito multidisciplinar que necessita do entendimento de áreas das engenharias mecânica, materiais e eletrônica. Formando assim, conhecimentos combinados nas áreas de monitoramento baseado em condições; tecnologias de evolução não destrutiva; novos métodos de modelamentos, e sensores de novas tecnologias (Gopalakrishnan et al., 2011). A Figura 1 mostra os elementos de combinação do SHM.

Figura 1 - Elementos de Combinação do SHM.

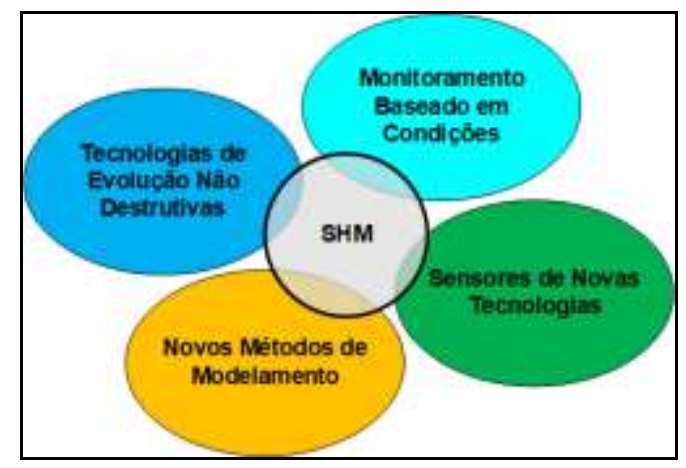

Fonte: Adaptado GOPALAKRISHNAN (2011), p 6.

O projeto de SHM, que visa o monitoramento e análise de integridade do sistema, deve conter elementos de hardware, compostos por sensores e instrumentos associados, e softwares, compostos por algoritmos de tecnologia conceitual matemática e física, que permitam a separação de grupos, seleção de informações e caracterização dos mesmos (Gopalakrishnan et al., 2011). A análise de integridade do sistema, na visão conceitual do SHM, consiste na observação de uma estrutura ou sistema 
mecânico ao longo do tempo, com atividades de medições periódicas e intervaladas, captadas por sensores, cujos dados extraídos são analisados utilizando métodos estatísticos para a determinação do estado da peça, caracterizando ou não os danos encontrados (Gopalakrishnan et al., 2011; Deraemaeker, 2010). Portanto, na integridade do sistema é feito um diagnóstico para determinar o estado da vida útil do componente, e posteriormente, um prognóstico quem envolve o grau de severidade do dano no componente (Gopalakrishnan et al., 2011).

A detecção dos danos ou variações no sistema dinâmico estrutural pode ser feita utilizando a teoria de vibrações, adotando um determinado critério na região de contorno, cujos modos de vibrar, frequência natural do sistema, podem ser detectados através do conjunto sensor, placa, software (Gopalakrishnan et al., 2011).

A análise de vibração atua diretamente na decisão de parada de máquina para a manutenção do equipamento, reparo, ou troca do componente, cuja decisão, é feita baseada nas características e variação das propriedades mecânicas do componente analisado (Yan, 2015). Os diferentes métodos de análise de sinal, aplicados a teoria de vibração, permitem que os resultados da prognose de danos e falhas sejam utilizados em segurança e confiabilidade estrutural (YAN, 2015). Na análise digital de sinais é possível introduzir conceitos estatísticos de testes de hipóteses para sistemas paramétricos e não paramétricos (Trivedi, 2016).

O SHM também utiliza métodos de simulação computacional conceituando as técnicas de elementos finitos, métodos de perturbações, entre outros, o qual analisa o modo espectral das ondas, para a detecção de danos e falhas em um determinado sistema dinâmico (Gopalakrishnan et al., 2011).

\section{Motores de Combustão - Princípios de Vibração e Acústica}

Os motores de ignição a faísca utilizam como fonte de alimentação de energia os cilindros, cuja energia da mistura dosada combustível-ar, ao entrar em combustão utilizando a faísca, transfere essa energia aos cilindros, e assim, os processos de admissão, compressão, explosão e escape ocorrem sequencialmente nos cilindros. Considerando o ciclo dos processos, os componentes mecânicos se movimentam, resultando no movimento frenético dos componentes. Assim, a frequência do movimento dos cilindros ocorre conforme a variação da mistura combustível-ar é alterada (Pulkrabek, 2003). Portanto, um sistema pode ser definido como a montagem dos componentes atuando conjuntamente como um todo, e quando uma força atua excitando o sistema (input), esse sistema demonstra uma resposta (output) (Meirovitch, 1997).

Através de um modelo físico é possível demonstrar matematicamente a correspondência entre as condições de input e output do sistema. A Figura 2 mostra a relação de correspondência e a resposta de excitação harmônica (Meirovitch, 1997).

Figura 2 - Input e Output da Função da Resposta em Frequência.

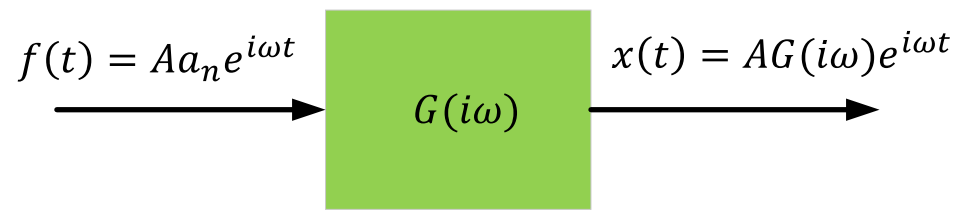

Fonte: Meirovitch (1997).

O input corresponde a equação $f(t)=A a_{n} e^{i \omega t}$ e o output corresponde a equação $x(t)=A G(i \omega) e^{i \omega t}$, o qual $a_{n}$ é um coeficiente de $x(t)$, e $e^{i \omega t}=\cos (\omega t)+i \operatorname{sen}(\omega t)$ é um vetor complexo. Note que, $G(i \omega)$ é a resposta em frequência das características do sistema dinâmico, assim, 


$$
G(i \omega)=\frac{a_{n}}{z(i \omega)}=\frac{a_{n}}{a_{0}(i \omega)^{n}+a_{1}(i \omega)^{n-1+\cdots+a_{n}}}
$$

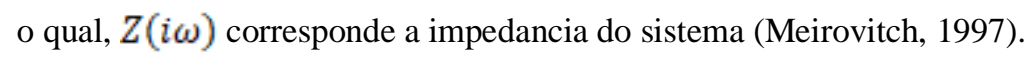

A vibração é considerada como a oscilação de um corpo qualquer que ocorre por um determinado tempo, cuja frequência de oscilação depende de fatores como o atrito inerente ao sistema, à massa e a rigidez do sistema em estudo (Ganguli \& Panchore, 2018).

Considerando que o sistema contenha uma massa, amortecimento e mola o sistema dinâmico harmônico pode ser demonstrado através do diagrama de corpo livre. A Figura 3 mostra o diagrama de corpo livre de um sistema dinâmico, que corresponde ao estudo de um sistema vibracional harmônico.

Figura 3 - Diagrama de Corpo Livre - Sistema Massa, Mola e Amortecimento.

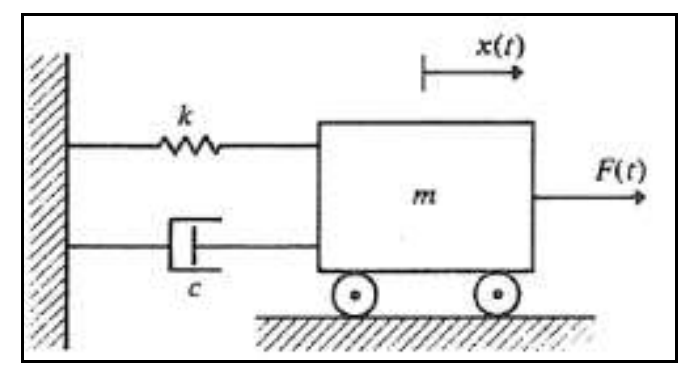

Fonte: Meirovitch (1997).

A equação geral do sistema pode ser escrita como,

$$
m \ddot{x}(t)+c \dot{x}(t)+k x(t)=f(t)
$$

o qual, $m$ é a massa, $c$ é o amortecimento, $k$ é a constante elástica e $f(t)$ é uma força qualquer. Note que $x(t)=A \cos \left(\omega_{n} t-\varphi\right)$, e $\omega_{n}=\sqrt{\frac{k}{m}}=\frac{2 \pi}{T}$ corresponde à frequência natural do sistema, onde $T$ é o período de oscilação. Considerando as condições iniciais e que $f(t)=0$, a resposta do sistema de single degree of freedom (SDOF), pode ser reescrita considerando que a equação geral do movimento seja dividida pela massa $m$, assim,

$$
\begin{aligned}
& \ddot{x}(t)+2 \zeta \omega_{n} \dot{x}(t)+\omega_{n}^{2} x(t)=0 \\
& \zeta=\frac{c}{2 m \omega_{n}}
\end{aligned}
$$

o qual $\zeta$ é o fator de amortecimento viscoso (Meirovitch, 1997; Crocker, 2007). Assim, a resposta em frequência do sistema harmônico, pode ser escrito como,

$$
G(i \omega)=\frac{\omega_{n}^{\frac{n}{n}}}{(i \omega)^{2}+2 \zeta \omega_{n}(i \omega)+\omega_{n}^{2}}=\frac{1}{1-\left(\frac{\omega}{\omega_{n}}\right)^{2}+\frac{i 2 \zeta \omega}{\omega_{n}}}
$$


A resposta em frequência do sistema pela transformada de Laplace pode ser escrita como,

$$
X(s)=\frac{s+2 \zeta \omega_{n}}{s^{2}+2 \zeta \omega_{n} \omega^{s}+\omega_{n}^{2}} x_{0}+\frac{1}{s^{2}+2 \zeta \omega_{n} s+\omega_{n}^{2}} v_{0}
$$

o qual, $x_{0}$ é a posição no instante inicial; e $v_{0}$ é a primeira derivada de $x(t)$ (Meirovitch, 1997; Crocker, 2007).

A energia do choque sucessivo entre as moléculas alterna a densidade do meio, resultando na propagação desta energia por um determinado tempo a uma determinada distancia que é conhecido como um ruído sonoro ou onda acústica (Kinsler et al., 1999; Crocker, 2007). Considerando este fato, a oscilação dos componentes do sistema mecânico movimenta o meio fluídico ao redor, e assim, a transmissão do ruído sonoro se propaga a uma determinada distancia por um tempo determinado.

A onda linear acústica é formada pelo: princípio da equação do estado para gases, que relacionam temperatura, pressão, volume e entropia; princípio da equação da continuidade que determina o princípio da conservação de massa; e pelo princípio da equação de Euler. O resultado da aplicação dos três princípios resulta na equação da onda,

$$
\left(\frac{\partial^{2} p}{\partial t^{2}}\right)=c^{2}\left(\frac{\partial^{2} p}{\partial x^{2}}\right)
$$

sendo, $P$ é a pressão do meio; $t$ é o tempo decorrido; $c$ é a velocidade de propagação da onda, e $x$ é o deslocamento da onda no meio (Kinsler et al., 1999; Crocker, 2007).

\section{Análise e Processamento de Sinais}

Um sinal pode ser entendido como um pulso elétrico que contém informações do estado de um sistema, cuja comunicação é formada por códigos e podem ser analisadas por métodos de processamento. Os sinais possuem relações de dependência com o tempo, e são divididos em tempo contínuo ou tempo discreto. Portanto, o sinal no tempo discreto é definido em instantes periódicos, e no tempo contínuo é definido a qualquer instante. Os sinais digitais são compostos de valores discretos (valores possíveis), e os analógicos compostos de qualquer valor. A análise digital de sinal tende a resolver diversos problemas utilizando métodos como a análise espectral, filtros de frequência, entre outros, e são aplicados em diferentes indústrias para busca de resultados e redução de custos (Hussain et al., 2011).

A representação matemática do sinal no domínio do tempo pode ser demonstrada pela convolução da reposta ao impulso $h(t)$ e o sinal de entrada $x(t)$, assim,

$$
y(y)=h(t) * x(t)=\int_{-\infty}^{\infty} h(\lambda) x(t-\lambda) d \lambda
$$

O sinal no domínio da frequência pode ser analisado utilizando a transformada de Fourier (FT) e a transformada de Laplace (LT) (Oppenheim et al., 1998; Hussain et al., 2011).

\subsection{Filtro de Wiener}

O filtro de Wiener foi desenvolvido por Norbert Wiener por volta de 1940, otimizando um sinal enviado pelo conhecimento do espectro do sinal enviado com o recebido. A função de transferência otimizada pode ser escrita como, 


$$
H_{0}(s)=\frac{s_{y x(s)}}{s_{x x}(s)} e^{\alpha s}
$$

o qual, $\alpha$ é o atraso do filtro de Wiener $H_{0}(s) ; S_{x x}(s)$ é a função de densidade espectral (PSD) do sinal original; e $S_{y x}(s)$ é a densidade da correlação espectral (CSD) entre o sinal original e o observado. A Figura 4 mostra o modelo linear genérico estimado do filtro de Wiener (Hussain et al., 2011; Levy, 2020).

Figura 4 - Modelo Genérico Linear Estimado do Filtro de Wiener.

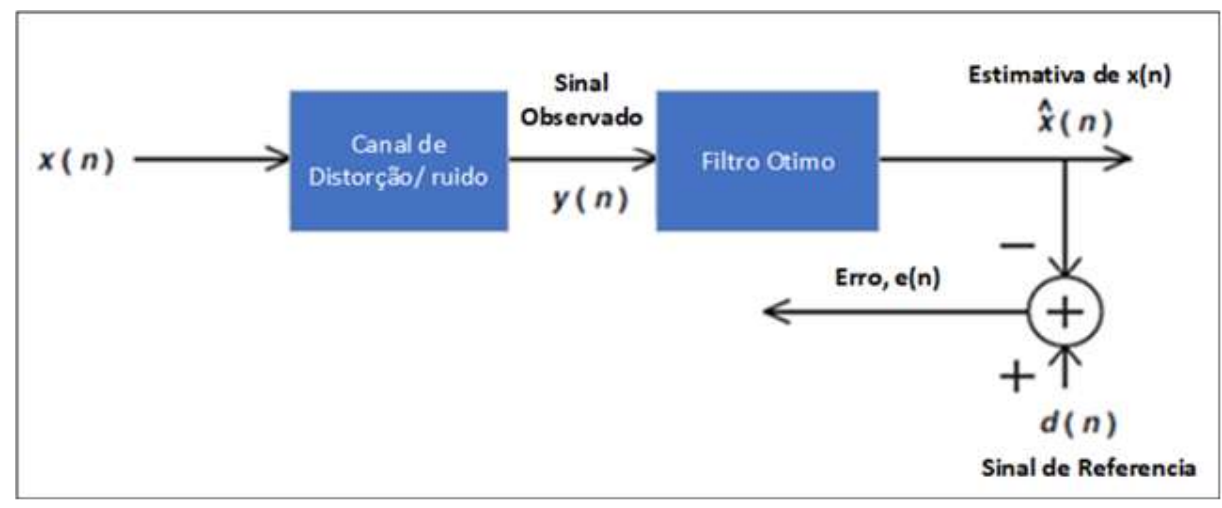

Fonte: Hussain et al. (2011), p.157.

A equação do filtro de Wiener pode ser reescrita considerando a minimização do erro da raiz quadrada entre o sinal observado $y(n)$ e o sinal de referência $d(n)$, que corresponde ao sinal original $x(n)$ (Hussain et al., 2011; Levy, 2020). Assim, a equação é reescrita como,

$$
h_{o p t}=\left[R_{y y}^{-1} R_{y d}\right]^{T}
$$

\subsection{Sistema imunológico artificial}

O sistema imunológico tem como capacidade, reconhecer e estimular as diferenças de mecanismos próprios e nãopróprios (self-nonself), cuja precisão da resposta permite que o conceito seja aplicado na construção de modelos de inteligência artificial, conhecido como sistemas imunológicos artificiais. Esses sistemas ou modelos de inteligências atuam diretamente nas funções de reconhecimento de padrões, aprendizado e aquisição de memória, tolerância e detecção de falhas, e diagnóstico de falhas, podendo ser aplicados diretamente na área de engenharia (Dasgupta \& Niño, 2009; Tan, 2016).

Basicamente, o sistema imunológico biológico (BIS) determina o fluxo básico para o desenvolvimento do algoritmo utilizando as funções de aprendizado e memória. Portanto, o algoritmo do sistema imunológico artificial (AIS) pode ser desenvolvido utilizando um critério geral: o antígeno corresponde a função objetivo e resolve problemas e restrições; o anticorpo corresponde a solução candidata; o grau de correspondência de afinidade de antígeno e anticorpo, corresponde a solução candidata com função objetiva (Dasgupta \& Niño, 2009; Tan, 2016). A Figura 5 mostra a etapas do AIS para o desenvolvimento do algoritmo. 
Figura 5 - Etapas de Desenvolvimento do Algoritmo AIS.

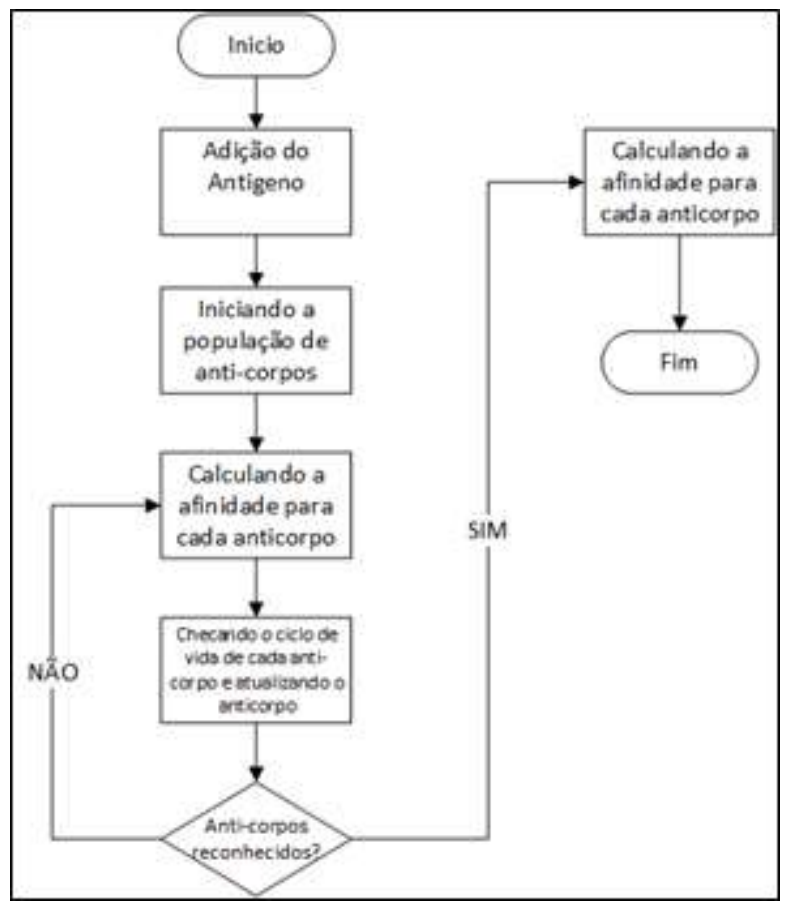

Fonte: Tan (2016).

O sistema imunológico biológico contém diferentes teorias, propostas por imunologistas, pois possui a capacidade de aprendizado contínuo e de adaptação. Com isso, diversos modelos foram desenvolvidos, sendo, a teoria de seleção clonal, modelo de rede imune e teoria do perigo (Dasgupta \& Niño, 2009; Tan, 2016). A seleção negativa é considerada um processo de reconhecimento de um ligante por um receptor celular, cujo processo de interação entre o linfócito e o antígeno pode resultar na deleção clonal (morte) ou anergia clonal (anergia) deste linfócito (De Castro, 2007).

Baseado no sistema imunológico biológico de seleção negativa, Forrest et al. (1994), propõe um algoritmo de seleção negativa que detecta anomalias em sistemas de computador, utilizando o princípio de que, são gerados detectores por seleção negativa que reconhecem quem invade ou não o computador. O método é distinguir as células próprias e não próprias, ou seja, reconhecer as moléculas que são do organismo (próprias), das moléculas estranhas (não próprias). O algoritmo do sistema imunológico artificial de seleção negativa possui duas fases: a primeira é considerada o gerador de detectores; a segunda é considerada o monitoramento dos dados protegidos comparando com os detectores, considerando que, se um detector for ativado sabe-se que a mudança ocorreu. A Figura 6 mostra a geração de detectores, considerado censoriamento, e a Figura 7 mostra o monitoramento dos caracteres protegidos para as alterações (Forrest et al., 1994). 
Figura 6 - Sensoreamento (Geração de Detectores).

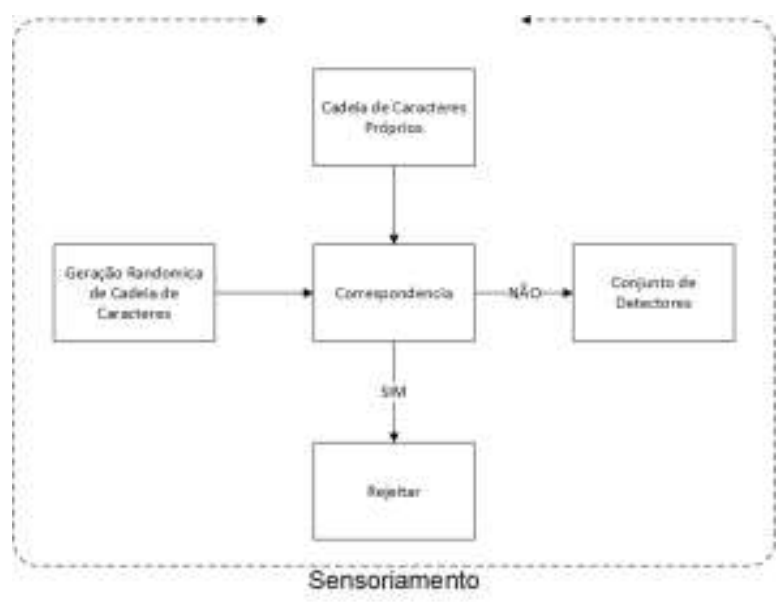

Fonte: Forrest et al. (1994).

Figura 7 - Monitoramento (Monitoramento dos Dados).

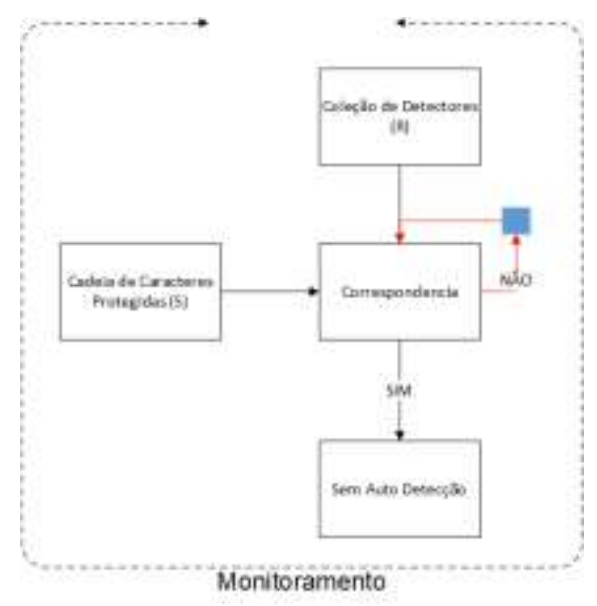

Fonte: Forrest et al. (1994).

O conceito de correspondência (match) perfeita entre duas cadeias (strings) de tamanhos iguais significa que cada local da cadeia (string) contém símbolos idênticos, porém, este caso é considerado extremamente raro, e assim, é necessário introduzir o conceito de correspondência parcial. A regra da correspondência parcial é aplicada quando $r$ correspondências próximas tem símbolos e posições correspondentes, assim, duas cadeias quaisquer $(x, y)$ são correspondentes (verdadeiras) quando $x$ e $y$, correspondem pelo menos a $r$ locais próximos. A probabilidade $\left(P_{m}\right)$ de duas cadeias quaisquer se corresponderem pode ser calculada pela equação,

$$
P_{m} \approx\left(\frac{\left(\frac{(1-r)(m-1)}{m+1}\right)}{m^{r}}\right)
$$

$l$ corresponde ao número de símbolos da cadeia (comprimento); $m$ corresponde ao número de símbolos do alfabeto; e r é o número de correspondência próxima necessárias para ocorrer a correspondência (Forrest et al., 1994). 
Considerando que, a correspondência de proximidade seja determinada $(r)$, é introduzido o conceito da taxa de afinidade das cadeias $\left(T_{a f f}\right)$, que determina o grau de semelhança necessário para que se ocorra a correspondência. Esta condição pode ser descrita, considerando a equação,

$$
T_{a f}=\left(\frac{A_{n}}{A_{\mathrm{t}}}\right) 100
$$

sendo $A_{n}$ corresponde ao número de cadeias normais no problema (própria), $A_{t}$ corresponde ao número total de cadeias do problema (cadeias próprias e não-próprias) (Bradley, et al., 2002).

A quantificação da afinidade dos padrões analisados $\left(Q_{a f}\right)$, pode ser definido matematicamente pela equação,

$$
Q_{a f}=\frac{\sum_{i=1}^{L} V_{c}}{L} 100
$$

o qual $V_{c}$ corresponde as variáveis correspondentes; $L$ corresponde a quantidade total de variáveis; $\sum_{i=1}^{L} V_{\varepsilon}$ corresponde a somatória das variáveis correspondentes. Assim, para que ocorra a correspondência com os padrões deve-se existir a condição $Q_{a f} \geq T_{a f}$ (Lima et al, 2013).

\section{Metodologia}

Este trabalho foi desenvolvido internamente a um laboratório ainda em construção, porém, foram consideradas as normas de segurança vigentes para a experimentação.

O experimento consiste em um motor de motocicleta posicionado sobre uma bancada de teste fixada ao solo. No motor foi posicionado um acelerômetro MPU6050 ligado a uma placa de arduino UNO, o qual está ligado ao computador para a transmissão dos dados via USB. No computador um algoritmo desenvolvido em python faz a leitura do sinal e armazena em um banco de dados.

Os equipamentos deste trabalho são: um motor de 150cc, à gasolina; combustível gasolina e etanol; bancada de fixação do motor; acelerômetro MPU6050; placa de arduino UNO; computador; cabos e conexões. O objetivo do experimento é demonstrar o funcionamento do motor com combustível de posto, aplicando a teoria de vibração e a teoria de análise digital de sinais com seus respectivos conceitos. A Figura 8 mostra o motor com os respectivos equipamentos e suas ligações. Este experimento está dividido em duas fases de processamento, sendo o primeiro, relativo à medição do motor somente com a gasolina do posto sem qualquer acréscimo de outro combustível. Isso é necessário para a formação de um banco de dados padrão do experimento. A segunda fase se dedica a coletar sinais de misturas de combustíveis. 
Figura 8 - Motor acoplado a bancada com os respectivos.

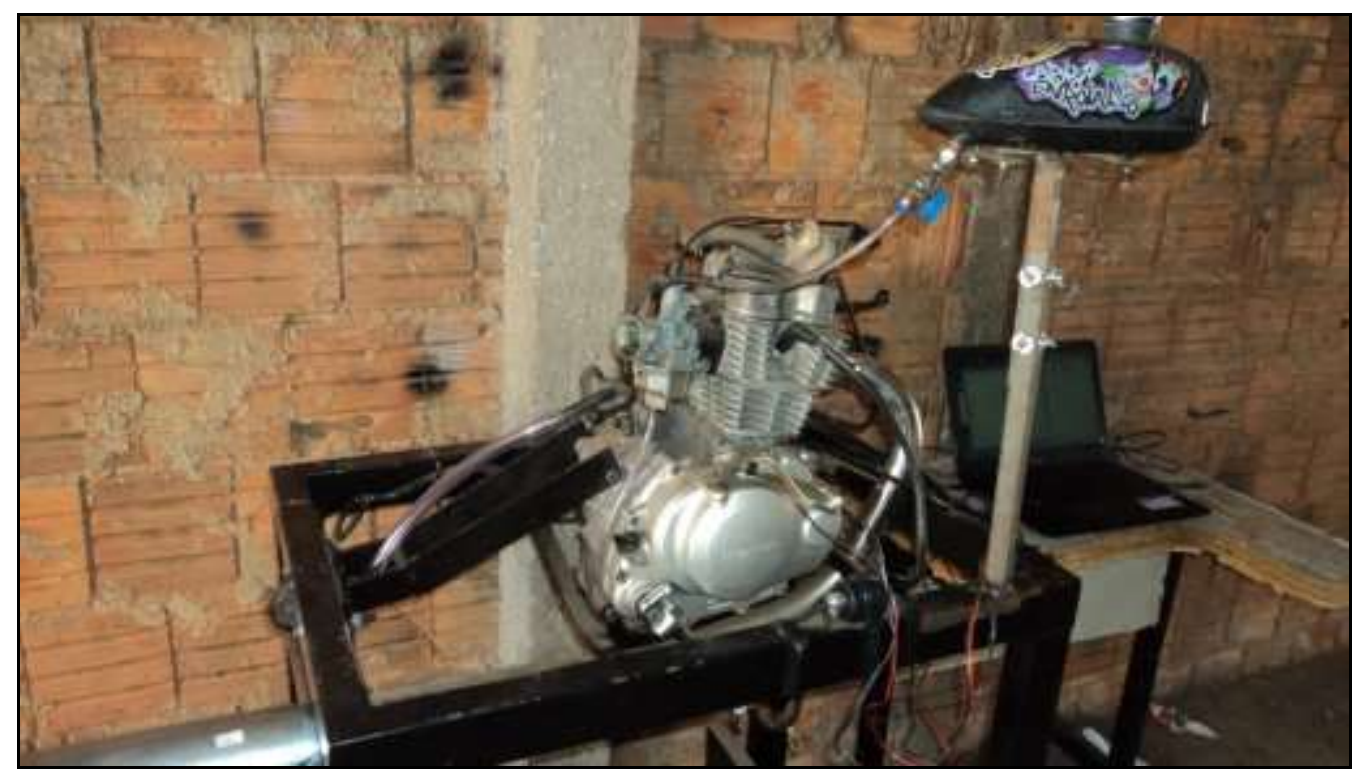

Fonte: Desenvolvimento Próprio.

A Figura 9 mostra o detalhe do acoplamento do sensor no motor.

Figura 9 - Detalhe de fixação do sensor no motor.

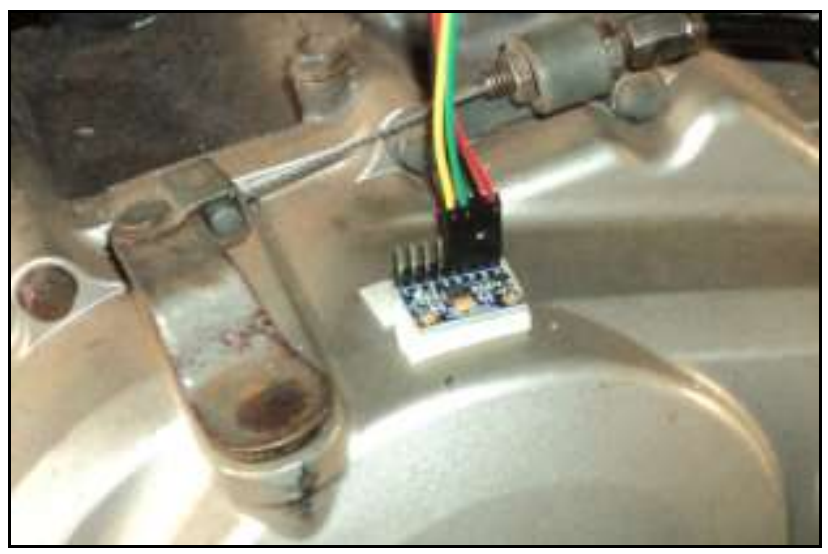

Fonte: Desenvolvimento Próprio.

A fixação do sensor foi feita utilizando uma fita dupla face sobre a estrutura da caixa de câmbio. A experimentação consiste em acionar o motor e, após um tempo curto, iniciar a medição sem alterar a rotação, mantendo sempre sem o engrenamento das marchas.

Através deste tipo de configuração experimental, é possível demonstrar a vibração do motor com o combustível e, caso exista algum tipo de ruído intrínseco ao sistema, é possível filtrá-lo no sinal utilizando o processamento pelo método de filtro de Wiener. O banco de dados desta primeira fase é composto de 10 sinais de tamanho 1x4000. Utilizando o teorema de cálculo amostral finito, para a análise de 4000 dados amostrais, o erro amostral é de $2 \%$ com base de confiança de $95 \%$, o qual seria necessário o tamanho de amostra de 1111 amostras.

A Figura 10 mostra o resultado do sinal médio de vibração. 
Figura 10 - Sinal Médio de Vibração da Gasolina do Posto de Combustível.

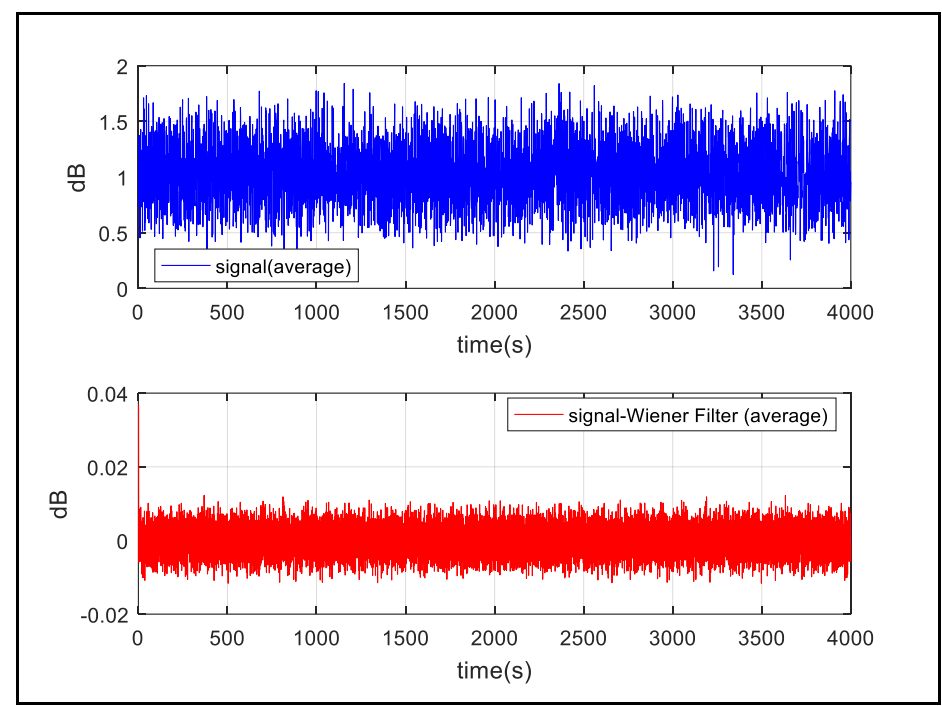

Fonte: Desenvolvimento Próprio.

Note que o sinal de vibração do motor utilizando a gasolina de posto de combustível em azul, é o sinal sem filtro, e o sinal em vermelho é o sinal já filtrado pelo filtro de Wiener. A Figura 11 mostra o sinal sem o filtro e o sinal filtrado no domínio da frequência.

Figura 11 - Sinal de Vibração no domínio da frequência.

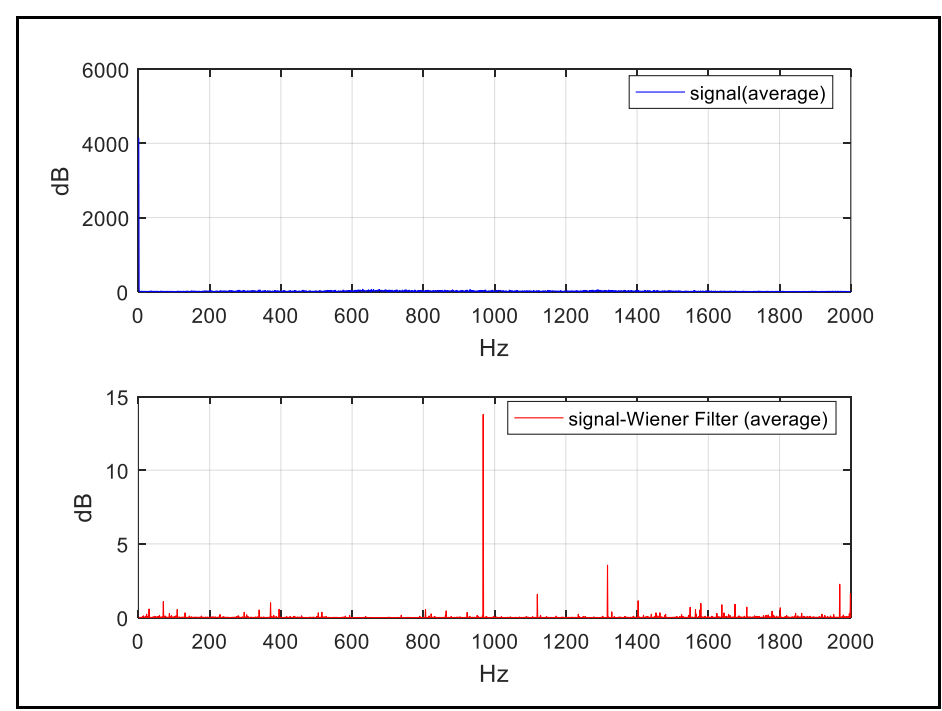

Fonte: Desenvolvimento Próprio.

A segunda fase do experimento tem como objetivo entender o comportamento do motor com diferentes proporções de combustíveis. O combustível inserido no motor é composto de:

A. gasolina de posto a $100 \%$, inserindo $100 \mathrm{ml}$ de etanol;

B. gasolina de posto a $100 \%$, inserindo $200 \mathrm{ml}$ de etanol.

Considerando que a mistura é de $500 \mathrm{ml}$ de gasolina de posto e $100 \mathrm{ml}$ e $200 \mathrm{ml}$ de etanol, respectivamente, para cada experimentação está sendo introduzidos $20 \%$ e $40 \%$ de etanol no combustível principal gasolina de posto. A Figura 12 mostra o resultado da aplicação do algoritmo do sistema imunológico artificial (AIS) no banco de dados A. 
Figura 12 - Resultado da classificação e grupamento do sinal do banco de dados A.

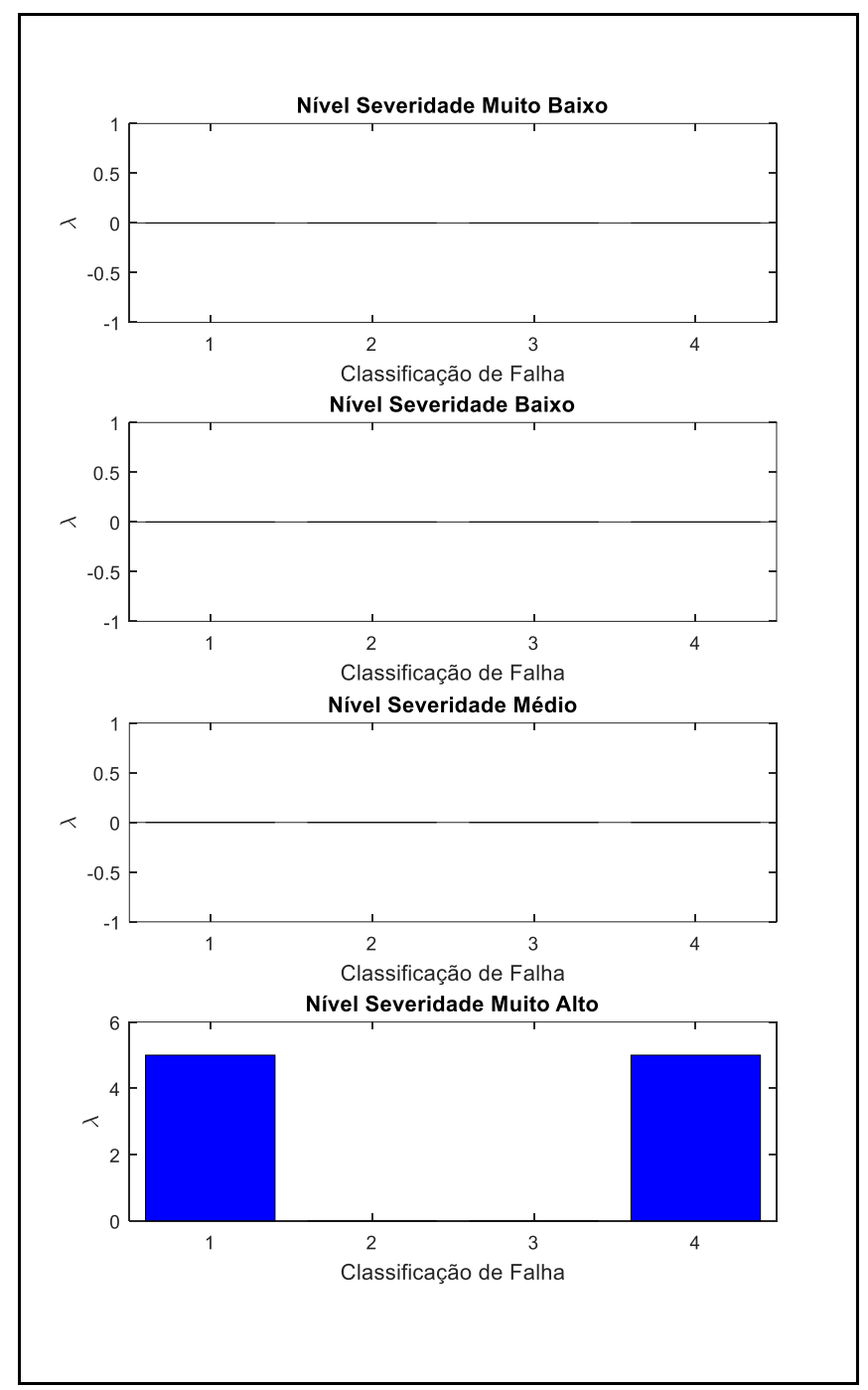

Fonte: Desenvolvimento Próprio.

No gráfico, o eixo x corresponde a probabilidade das falhas, sendo distribuído da seguinte forma, (1) probabilidade de falha muito baixa; (2) probabilidade de falha baixa; (3) probabilidade de falha moderada; (4) probabilidade de falha alta. No eixo y, é demonstrada a quantidade de sinais correspondentes à separação e grupamento do banco de dados. O algoritmo separa ainda os sinais pelo nível de severidade de falhas, sendo eles, severidade muito baixa; baixa; média; e alta. O nível de severidade corresponde ao grupamento de sinais correspondente a locação vertical internamente a disposição do gráfico.

Através destas informações, note que, no banco de dados A (gasolina de posto a 100\%, inserindo $100 \mathrm{ml}$ de etanol), os sinais foram agrupados e classificados quanto no nível de severidade da falha muito alto, demonstrando que 4 sinais (1x4000) correspondem a probabilidade de falha muito baixa, e 6 sinais (1x4000) na probabilidade de falhas alta. Esta separação dos sinais corresponde diretamente à forma de vibrar do sistema, cuja vibração do motor, demonstrou oscilações para este tipo de combustível misturado. A Figura 13 mostra a aplicação do algoritmo do AIS no banco de dados B. 
Figura 13 - Resultado da classificação e grupamento do sinal do banco de dados B.

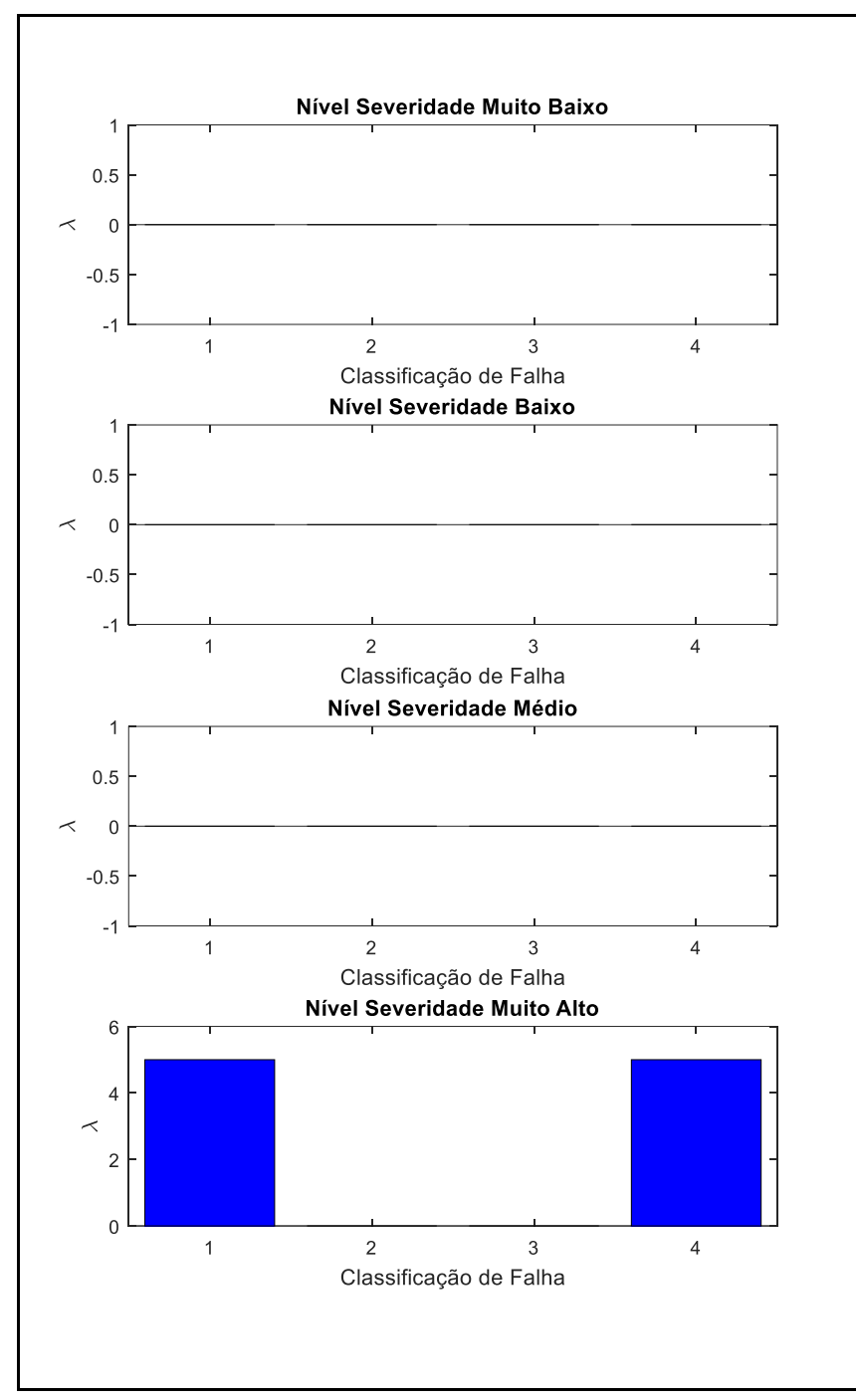

Fonte: Desenvolvimento Próprio.

No banco de dados B (gasolina de posto a 100\%, inserindo $200 \mathrm{ml}$ de etanol), os sinais foram agrupados e classificados quanto a nível de severidade da falha muito alto, demonstrando que 5 sinais (1x4000) correspondem a probabilidade de falha muito baixa, e 5 sinais $(1 \mathrm{x} 4000)$ na probabilidade de falhas alta. Ocasionados também pela forma de vibrar do motor. A Figura 14 mostra a aplicação do algoritmo do AIS no banco de dados A e B. 
Figura 14 - Resultado da classificação e grupamento do sinal do banco de dados A e B.

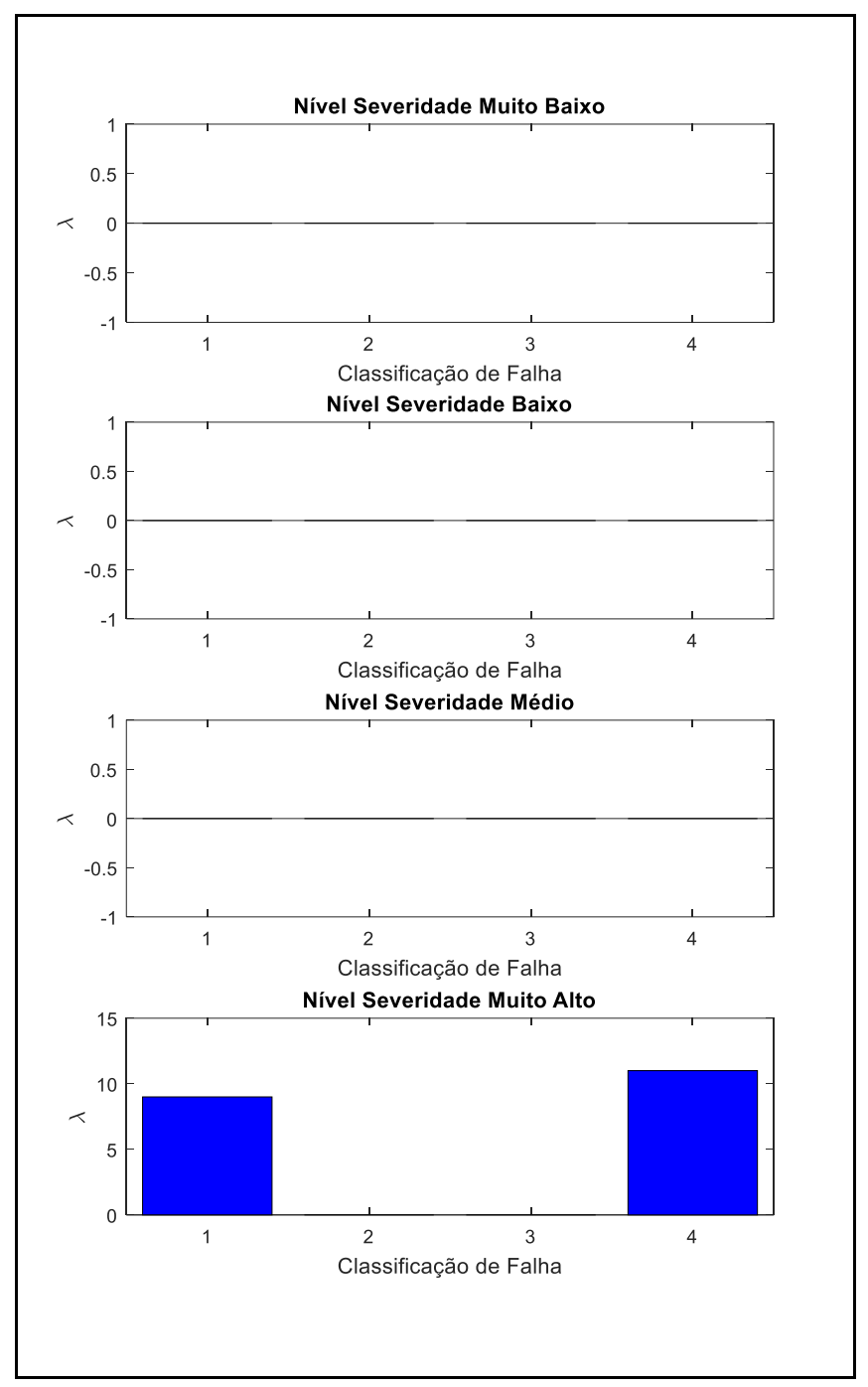

Fonte: Desenvolvimento Próprio.

Note que, a aplicação do AIS no banco de dados A e B, demonstra que os 20 sinais, foram distribuídos de forma: 9 sinais no grupo de probabilidade de falha muito baixo; e 11 sinais no grupo de sinais de probabilidade de falha alta. Neste aspecto, a formação do conceito se manteve, considerando que, todos os sinais se enquadram em um nível de severidade alto.

O resultado quantitativo e qualitativo dos bancos de dados do sinal do combustível de posto gasolina (sinal de referência), do sinal de combustível de posto com 100ml de etanol, e do sinal de combustível de posto com 200ml de etanol, podem ser demonstrados nos gráficos de regressão linear. 
Figura 15 - Regressão Linear sinal de combustível de posto com sinal de combustível de posto com 100ml de etanol.

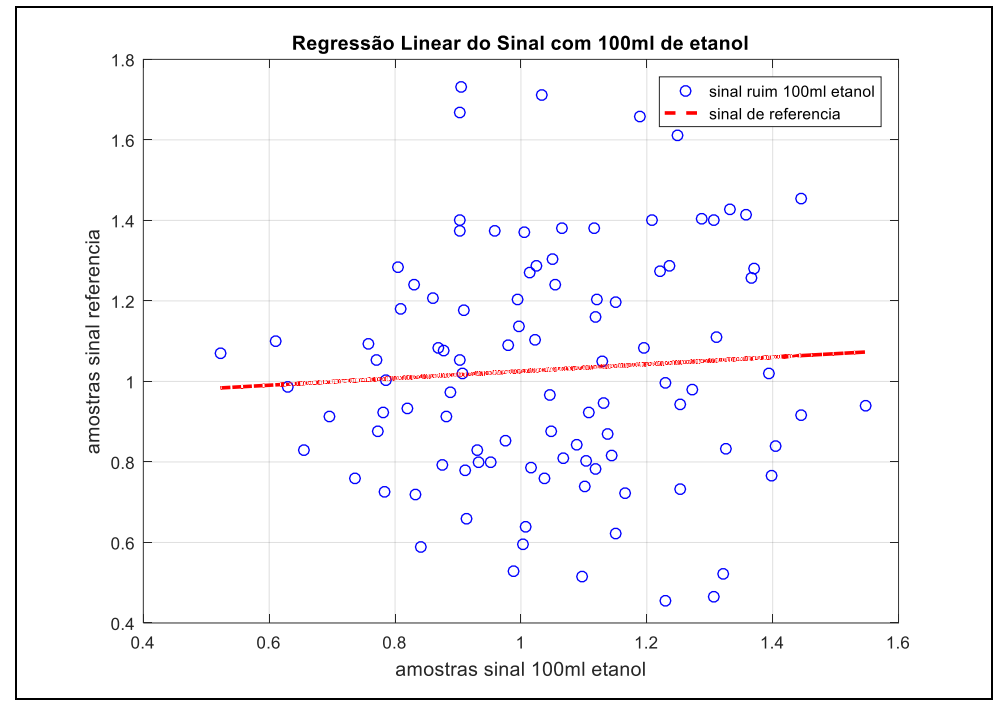

Fonte: Desenvolvimento Próprio.

A figura demonstra que a distribuição dos valores do sinal ruim está distante e desalinhada em relação ao a reta de melhor ajuste. Considerando a equação da reta $(\mathrm{ax}+\mathrm{b})$, o argumento a = 0.087070, e o argumento b é 0.938312 .

Figura 16 - Regressão Linear sinal de combustível de posto com sinal de combustível de posto com $200 \mathrm{ml}$ de etanol.

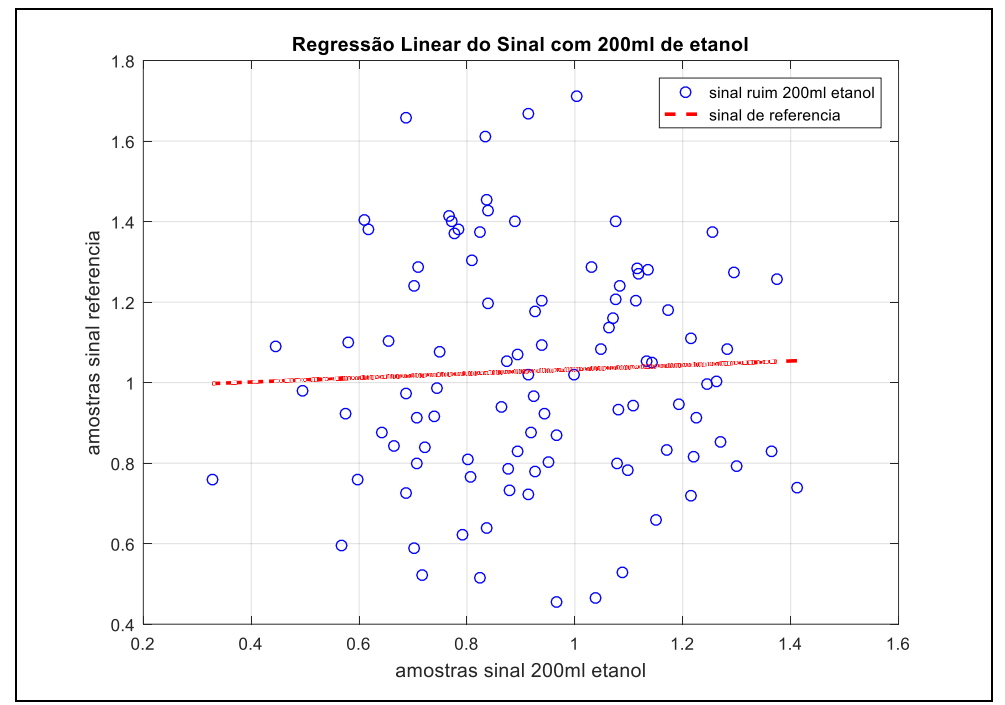

Fonte: Desenvolvimento Próprio.

A figura demonstra que a distribuição dos valores do sinal ruim está distante e desalinhada em relação ao a reta de melhor ajuste. Considerando a equação da reta $(\mathrm{ax}+\mathrm{b})$, o argumento a $=0.052345$, e o argumento b é 0.980550 .

Note que em ambos os casos a dispersão do sinal do combustível de posto de combustível com etanol de $100 \mathrm{ml}$ e 200ml, estão dispersos em relação a reta de melhor ajuste.

\section{Discussão}

O filtro de Wiener, ao ser aplicado no sinal original com ruídos, demonstrou a eliminação dos ruídos, o que possibilita a melhora da qualidade para aplicações no AIS. O banco de dados A e B, ao serem analisados pelo algoritmo de AIS, 
demonstraram que dos $100 \%$ dos sinais analisados a taxa de afinidade entre os sinais é de $70 \%$, que é a relação entre o processamento e o conhecimento dos sinais não próprios.

Observa-se ainda que a distinção dos sinais com quantidades de etanol de $100 \mathrm{ml}$ e $200 \mathrm{ml}$ na gasolina em relação a reta de melhor ajuste, também podem ser vistos nos gráficos de regressão linear. Isto demonstra que a técnica utilizada com o AIS de seleção negativa para detecção das diferenças entre os sinais de vibração é precisa, e ainda demonstra a classificação do sinal do combustível quanto ao grau de severidade.

Nota-se, portanto, que é possível de se reconhecer e separar os sinais do motor de cada tipo de mistura de combustível através da técnica de AIS de seleção negativa.

\section{Conclusão}

Este trabalho demonstra a aplicação de um conceito multidisciplinar separando-o em duas fases distintas, sendo a primeira fase, a aplicação do filtro de Wiener nos bancos de dados do sinal com combustível de posto, A e B; e a segunda fase, a aplicação do AIS no banco de dados A e B, propiciando a separação e classificação dos sinais pelas falhas do sistema dinâmico.

Observou-se que o algoritmo é robusto e tende a detectar e classificar os combustíveis misturados pela teoria de vibração, perfazendo a ideia de que, cada combustível tem a sua forma de vibrar.

A aplicação deste método promove uma categoria de análise em que, para cada tipo de vibração do motor, pode ser correspondida a explosão da mistura, o qual se relaciona com princípios da mecânica dos fluidos e da termodinâmica. Com isso, tipos de estudos de propriedades físico-químicas relacionados à análise da mistura, podem ser minimizados.

Considerando os resultados deste trabalho é possível afirmar que este trabalho está concluso e tem um resultado de sucesso, onde o método, pode ser aplicado por indústrias relacionados a área.

\section{Agradecimentos}

Os autores agradecem ao Centro Paula Souza - CPS, pelo apoio e incentivo à pesquisa nas FATEC's. Agradecemos ao Laboratório de Sistemas Complexos (Sisplexos) do Instituto de Química - UNESP, por ceder o espaço físico, o parque computacional e ao suporte financeiro da Fundação de Amparo à Pesquisa do Estado de São Paulo (FAPESP), através do processo 2019/10515-4 e ao Conselho Nacional de Desenvolvimento Científico e Tecnológico (CNPq), através do processo 312972/2019-9. Agradecemos ao Instituto de Pesquisas Tecnológicas do Estado de São Paulo S.A - IPT por permitir a parceria com a Fatec e viabilizar a capacitação da equipe técnica.

\section{Referências}

Bein, T., Elliott, S., Ferralli, L., Casella, M., Meschke, J., Saemann, E. U., Nielsen, F. K., \& Kropph, W., Integrated Solutions for Noise \& Vibration Control in Vehicles, Procedia - Social and Behavioral Sciences, 48, 919-931, 2012.

Bradley, D. W., \& Tyrrell, A. M., Immunotronics-novel finite-state-machine architectures with built-in self-test using self-nonself differentiation. IEEE Transactions on Evolutionary Computation, 6, 227-238, 2002.

Crocker, M. J, Handbook of Noise and Vibration Control, John Wiley \& Sons, 2007. 1569p.

Dasgupta, D., \& Niño, L. F. Immunological Computation: Theory and Applications, Taylor \& Francis Group, 2009. 298p.

De Castro, L. N., Fundamentals of Natural Computing: Basic Concepts, Algorithms, and Applications, CRC Press, 2007. 674p.

Deraemaeker, A., \& Worden, K. New Trends in Vibration Based Structural Health Monitoring, SpringerWien, 2010. 311p.

Farrar, C. R., \& Worden, K. Structural Health Monitoring: A Machine Learning Perspective, John Wiley, 2013. 643p. 
Research, Society and Development, v. 10, n. 4, e5110413741, 2021

(CC BY 4.0) | ISSN 2525-3409 | DOI: http://dx.doi.org/10.33448/rsd-v10i4.13741

Forrest, S., Perelson, A. S., Allen, L., \& Cheukuri, R., Self-Nonself Discrimination in a Computer, IEEE Computer Society Symposium on Research in Security and Privacy, 1994. 10.1109/RISP.1994.296580.

Gabbert, U., Duvigneau, F., \& Ringwelski, S., Noise Control of Vehicle Drive Systems, Facta Universitatis - Mechanical Engineering Series, 15(2), 183-220, 2017. 10.22190/FUME170615009G.

Ganguli, R., \& Panchore, V., The Rotating Beam Problem in Helicopter Dynamics, Springer Nature Singapore, 2018. 109p.

Gopalakrishnan, S., Ruzzene, M., \& Hanagud, S.; Computational Techniques for Structural Health Monitoring, Springer-Verlag, 2011. 517p.

Hussain, Z. M., Sadik, A. Z., \& O’Shea, P., Digital Signal Processing: An Introduction with MATLAB and Applications, Springer-Verlag, 2011. 373p.

James, F., Statistical Methods in Experimental Physics, World Scientific Publishing, 2006. 362p.

Levy, B. C. Random Processes with Applications to Circuits and Communications, Springer Nature, 2020. 467p.

Lima, F. P. A., Lotufo, A. D. P., \& Minussi, C. R. Artificial Immune Systems Applied to Voltage Disturbance Diagnosis in Distribution Electrical Systems, Proceedings on IEEE PowerTech-2013, 2013. 10.1109/PTC.2013.6652127.

Meirovitch, L., Principles and Techniques of Vibrations, Prentice-Hall, 1997. 694p.

Montgomery, D. C., \& Runger, G. C., Applied Statistics and Probability for Engineers, (3a ed.,) John Wiley \& Sons, 2003. 978p.

Oppenheim, A. V., Schafer, R. W., \& Buck, J. R. Discrete Time Signal Processing, Prentice Hall, 1998. 897p.

Outa, R., Chavarette, F. R., Mishra, V. N., Gonçalves, A. C., Roefero, L. G. P., \& Moro, T. C., Prognosis and Fail Detection in a Dynamic Rotor Using Artificial Immunological System, Engineering Computations, 2020. 10.1108/EC-08-2019-0351.

Pulkrabek, W., Engineering Fundamentals of the Internal Combustion Engine, (2a ed.,) Pearson, 2003. 504p.

Roe, B. P., Probability and Statistics in Experimental Physics, (2a ed.,) Springer-Verlag, 2001. 262p.

Sacomano, J. B., Gonçalves, R. F., Da Silva, M. T., Bonila, S. H., \& Sátyro, W. C., Industria 4.0: Conceitos e Fundamentos, Editora Blucher, 2018.182p.

Tan, Y. Anti-Spam Techniques Based on Artificial Immune System, CRC Press, 2016. 264p.

Trivedi, K. S. Probability and Statistics with Reliability, Queuing, and Computer Science Applications, John Wiley \& Sons, 2016. 867p.

Yan, J. Machinery Prognostics and Prognosis Oriented Maintenance Management, John Wiley \& Sons, 2015. 356p. 